\title{
E-books didáticos nos ambientes de aprendizagem
}

\section{em rede}

\author{
Charles Rodrigues \\ Doutorando; Universidade Federal de Santa Catarina (UFSC); \\ falecomcharles@yahoo.com.br \\ Richard Perassi \\ Doutor; Universidade Federal de Santa Catarina (UFSC); \\ richard.perassi@uol.com.br \\ Bárbara Zardo De Nardi \\ Mestranda; Universidade Federal de Santa Catarina (UFSC); \\ babizardo@gmail.com \\ Francisco Antonio Pereira Fialho \\ Doutor; Universidade Federal de Santa Catarina (UFSC); \\ fapfialho@gmail.com
}

\begin{abstract}
Resumo: Apresenta-se um estudo sobre a relação entre os e-books e o ambiente virtual de aprendizagem (AVA) suportado por dispositivos eletrônicos conectados à Internet. Objetiva-se evidenciar as possibilidades e vantagens que justificam o interesse pelo uso de e-books como parte dos investimentos nos processos educativos em ambientes de aprendizagem em rede. Como metodologia, fez-se uma pesquisa bibliográfica de caráter exploratório com viés qualitativo. Como possibilidades e vantagens do uso de e-books didáticos, elencaram-se: a adaptação ou a personalização de processos interativos e conteúdos, conforme as características individuais do estudante; a amplitude no tempo e no espaço da comunicação e da interação entre estudantes e outros envolvidos; a eficiência e a agilidade na gestão do processo educativo; e a redução dos custos pessoais, financeiros e ambientais. Porém, para isso, depende-se do atendimento às necessidades de infraestrutura física e lógica, isto é, Internet de banda larga, rede de energia, mobiliários e equipamentos adequados.
\end{abstract}

Palavras-chave: E-books didáticos. Ambiente Virtual de Aprendizagem. Tecnologia educacional. E-learning.

\section{Introdução}

Em decorrência do desenvolvimento da tecnologia de informação e comunicação (TIC), o modo como são usadas as tecnologias digitais tem alterado as práticas e o ambiente educacional. O aprimoramento dos dispositivos móveis ligados à Internet permitiu acesso e mobilidade no manuseio de suportes da informação educativa em 
diferentes formatos. Isso incluiu o formato eletronic book (e-book) que é acessado com o uso de e-readers ou softwares específicos para aplicativos móveis (app) usados na leitura de textos (UNESCO, 2013).

Nos países desenvolvidos, observa-se que é mais evidente a tendência de adoção dos e-books nas práticas educativas, o que provoca a transferência da dinâmica escolar baseada nos livros impressos para os conteúdos digitais. Além dos avanços tecnológicos, aprimorando a experiência de leitura em suportes eletrônicos, surgem novas abordagens para a conversão e a criação de livros didáticos, os quais se afastam da digitalização por escâner de textos já impressos para serem integrados em interfaces mais ricas em possibilidades, inclusive em relações hipertextuais e linguagem multimídia (GSMA, 2011).

Há expectativas de que a próxima geração tecnológica dos e-readers e dos dispositivos eletrônicos móveis permita maior socialização de estudos e aprendizagem. Assim, os conteúdos educativos poderão ser oferecidos com o apoio de diversificados recursos internos dos dispositivos, tais como: calculadora, gravador de voz, câmera, cronômetro, sistema de localização ou global positioning system (GPS), acelerômetro, bússola e diversos tipos de sensores, entre outros. É necessário, entretanto, distinguir o que é especificamente o livro e qual o campo de evolução do $e$-book, para que este seja, de algum modo, distinto dos outros materiais didáticos ou educativos, visto que existem múltiplos recursos digitais para promover experiências pedagógicas aos estudantes.

$\mathrm{O} e$-book com finalidade educativa é um produto que, ao seguir a tradição e o formato do livro impresso, deve garantir o registro organizado do conteúdo didático, mantendo-o da maneira exata como foi proposta pelo autor. Na mesma tradição, o conteúdo pode ser sintetizado em gráficos, referenciados em mapas ou ilustrados com outras imagens. Além disso, como nos livros tradicionais, o e-book também costuma oferecer informações adicionais, contidas em índices remissivos, notas de rodapé, glossários e outros.

Atualmente, é interessante identificar o conteúdo e os formatos que caracterizam o e-book como um produto específico, separando-o de outras informações e, ainda, de atividades que promovem a interação do estudante com o seu conteúdo. Assim, essa tecnologia - um dos produtos componentes do Ambiente 
Virtual de Aprendizagem (AVA) - é proposta como a parte que contém e comunica a informação fixa e permanente do conteúdo teórico educativo. Podem-se utilizar ainda hiperlinks ou vínculos no corpo do $e$-book para promover a relação entre o seu conteúdo específico e as demais informações, efeitos e recursos interativos do ambiente. As notas de rodapé do livro tradicional podem, por exemplo, ser substituídas por hiperlinks relacionados a conteúdos complementares disponíveis no ambiente (AVA). O mesmo pode acontecer com alguns termos gerais do glossário e com as atividades educativas que, anteriormente, eram parte do caderno de atividades.

Os e-books didáticos são reconhecidos em países desenvolvidos, na área de Educação, como recursos necessários e eficientes no processo de ensino e aprendizagem em ambientes pedagógicos virtuais. De acordo com os resultados de estudos recentes realizados nos Estados Unidos pelo Departamento de Educação e pela Associação Nacional de Treinamento e Simulação, a instrução baseada em tecnologia pode reduzir entre $30 \%$ e $80 \%$ o tempo necessário para os estudantes atingirem um objetivo de aprendizagem. Segundo a Federal Communications Commission (FCC, 2012) os estudantes que participaram de processos de aprendizagem online, em média, obtiveram índices de desempenho melhores em relação a outros que só participaram de processos presenciais.

Diante da contextualização exposta, o objetivo aqui é evidenciar as possibilidades e as vantagens que justificam o interesse pelo uso de $e$-books como parte dos investimentos nos processos educativos em ambientes de aprendizagem em rede. Essa questão central se desdobra em outras intenções subjacentes, como: (a) identificar as necessidades básicas de infraestrutura física e lógica nas escolas e em torno das comunidades escolares para o uso dessas tecnologias; e (b) investigar a necessidade de treinamento, formação e envolvimento de professores, estudantes e de toda a comunidade escolar no uso das ferramentas e dos conteúdos didáticos digitais disponíveis nos ambientes de aprendizagem em rede.

Dessa forma, baseando-se na questão geral deste estudo - Como evidenciar as possibilidades e as vantagens que justificam o interesse no uso de $e$-books como parte dos investimentos nos processos educativos em ambientes de aprendizagem em rede? -, pretende-se: (a) tratar das conceituações, das distinções e das relações 
eficientes entre o e-book didático e o ambiente virtual de aprendizagem (AVA), considerando-se, sobretudo, os recursos online decorrentes da conexão via Internet; (b) expor as possibilidades de armazenamento de conteúdo didático em repositórios denominados 'nas nuvens', além das possibilidades de composição, comunicação e interação educativa online; (c) discutir as necessidades básicas de infraestrutura física e lógica, de treinamento, formação e envolvimento de professores, estudantes e toda a comunidade escolar nesse processo; e (d) descrever as possiblidades e as vantagens decorrentes do uso de $e$-books didáticos vinculados a ambientes virtuais de aprendizagem.

Destaca-se que a apresentação dessas questões não ocorre de modo estanque, pois seu desenvolvimento se desdobra em forma de tramas que se entrecruzam ao longo do texto.

Este artigo está estruturado em quatros partes: esta introdução, na qual o estudo é contextualizado e os problemas e os objetivos propostos são expostos; a metodologia, onde são explicadas as etapas de desenvolvimento do estudo; a fundamentação conceitual, onde são apresentados os conceitos relacionados à temática, com referências a diferentes abordagens que proporcionam sustentação às discussões; e as considerações finais, parte na qual são feitos os últimos apontamentos.

\section{Metodologia}

O conteúdo apresentado neste artigo é parte dos estudos realizados no contexto da disciplina Fundamentos de Mídia e Conhecimento, do Programa de Pós-graduação em Engenharia e Gestão do Conhecimento (EGC/UFSC). Trata-se do resultado de uma pesquisa bibliográfica, que, conforme esclarecem Cervo, Bervian e Silva (2007, p. 60) "[...] procura explicar um problema a partir de referências teóricas publicadas em artigos, livros, dissertações e teses", e exploratória, de método qualitativo.

$\mathrm{Na}$ pesquisa exploratória, buscaram-se fontes de informação nas bases de dados da Coordenação de Aperfeiçoamento de Pessoal de Nível Superior (CAPES) sobre o processo atual de aplicação de recursos tecnológicos digitais nos sistemas formais de Educação. Também pesquisaram-se fontes teóricas sobre conceitos e 
processos relacionados à temática em estudo. O percurso exploratório possibilitou a identificação das fontes e a seleção das informações e dos conceitos usados na composição deste artigo. O método qualitativo contribuiu para melhor compreender o fenômeno enquanto processo social e permitiu, com isso, a integração e a visualização de seu contexto.

Durante a pesquisa, desenvolveram-se as seguintes etapas: (1) estudos exploratórios para reconhecimento inicial do fenômeno e dos temas relacionados; (2) revisão de literatura, com desenvolvimento de estudos teóricos para compreender o objeto de estudo em seu contexto cultural; (3) levantamento de informações nas fontes selecionadas, como realidade observada; (4) seleção e interpretação de amostra da realidade observada; e (5) utilização da referência teórica para interpretar as informações coletadas na realidade observada.

\section{Fundamentação conceitual}

Nesta seção, são apresentados e comentados os conceitos relacionados à temática em estudo, com referência a diferentes abordagens, tais como: abordagem estrutural, pedagógica, sociológica, política ou econômica, dentre outras.

\subsection{E-book no contexto tecnológico da atualidade}

A existência social de uma mídia requer uma estrutura capaz de realizar as seguintes funções básicas: (1) suportar a informação; (2) ajustar e manter a configuração da informação; e (3) atuar como canal de comunicação da informação ou conectar-se a um canal capaz de transmitir a informação (PERASSI; MENEGHEL, 2010).

Isso consolida a mídia social como sistema mediador que, necessariamente, reúne três elementos básicos: suporte, veículo e canal (PERASSI; MENEGHEL, 2010). No caso do e-book, o dispositivo eletrônico - computador de mesa ou pessoal, tablet e smartphone, dentre outros - serve de suporte e, também, é fonte de emissão e sistema de controle da luz. Portanto, a luz atua como veículo físico da informação e canal de comunicação para informar algo ao leitor que está postado em 
frente à tela videográfica do dispositivo eletrônico, qualquer que seja.

Um software ou programa de computação é o recurso que permite o dispositivo eletrônico receber ou "baixar" o e-book da Internet e apresentá-lo ao leitor nos formatos previstos. Procópio (2010) apresenta o e-book como mídia e considera o produto composto por três elementos integrados: (1) o dispositivo físico de leitura (hardware); (2) o programa de leitura, denominado de e-reader (software), e (3) o e-book propriamente dito, conteúdo editorial em um formato que, tradicionalmente, é reconhecido como livro.

Existe uma constante criação e utilização de formatos editoriais específicos da cultura digital. Há, igualmente, o esforço cultural para adaptar os formatos tradicionais dos produtos editoriais impressos, como livros, revistas e jornais, para os suportes eletrônicos de produtos digitais. O desafio é manter a identidade funcional, visual e social desses produtos e, também, incrementá-los, aproveitando os recursos multimídia dinâmicos e interativos oferecidos pela mídia digital conectada à Internet.

Além do escaneamento digital e da disponibilização dos produtos gráficos editoriais em websites na Internet, busca-se desenvolver programas ou softwares de editoração gráfica para integrar os formatos dos produtos impressos aos suportes eletrônicos, incrementando-os com os recursos característicos da hipermídia. Bottentuit e Coutinho (2007, p. 2) assinalam que tais recursos devem ser considerados como:

[...] pontos importantes no que diz respeito ao aspecto estético, gráfico e organizacional, ou seja, o tipo de letra deve ser o mais adequado, a quantidade do texto deve ser mais distribuída entre as páginas, o uso de cores e os contrastes obedecem a critérios.

Isso é proposto porque o e-book deve expressar as especificações que o tornam adequado para ser lido nos dispositivos eletrônicos. Além da utilização de recursos multimídia, como sons, gráficos e vídeos, e da interatividade por meio de exercícios, quizes e jogos, as peculiaridades e as oportunidades características do $e$ book garantem sua usabilidade e, assim, reduzem a necessidade ou o interesse do leitor em imprimir o conteúdo no suporte de papel. 
Na perspectiva histórica, Procópio (2010, p. 45) considera que Michael Hart (1947-2011) foi pioneiro na concepção do livro eletrônico. Em 1971, Hart fundou o Projeto Gutenberg, cuja missão era “[...] encorajar a criação e distribuição de livros eletrônicos [...]" e, por meio de parcerias, constituir uma biblioteca digital gratuita que disponibilizasse livros de domínio público. Em princípio, o escaneamento digital dos livros impressos foi considerado positivo, porque ampliou a capacidade de armazenamento do conteúdo e possibilitou o transporte de grande quantidade de informação literária em mídia física compacta, como os antigos disquetes e os atuais compact disk (CD) ou pen drives, além de ter permitido o envio dessa informação através da Internet. Isso ampliou a mobilidade dos livros e o acesso das pessoas ao conteúdo literário.

Bottentuit e Coutinho (2007) destacam que o conteúdo é a parte mais importante do livro digital, como fonte das informações que embasam o conhecimento interessante ao leitor. Por isso, o formato do livro impresso e, também, do e-book deve proteger o conteúdo, mantendo-o como foi originalmente desenvolvido pelos autores, que se responsabilizam pelo modo de ordenação do conteúdo e das informações expressas pelo produto. Afinal, o princípio básico do formato livro ou e-book é a integridade do conteúdo. Assim, o que é particular do livro não pode ser alterado e, nesse sentido, o leitor não é um interagente em relação ao produto.

Considera-se, entretanto, que “[...] o conhecimento não dispõe de expressão própria, necessita ser expresso por diversos sinais ou informações em mídia." (CAVALCANTE et al., 2013, p. 18). Portanto, por ser a parte física da informação, a mídia suporta e expressa o conteúdo, ao mesmo tempo em que suporta e veicula outras mensagens em formatos diferentes dos e-books. Por isso, é necessário um software especial, e-reader, como programa capaz de codificar a mensagem luminosa no formato de $e$-book.

O software e-reader garante a integridade do conteúdo autoral do e-book e estabelece os vínculos ou hiperlinks, os quais permitem a comunicação e a interação do leitor e do interagente com outros recursos e informações disponíveis no acervo do dispositivo eletrônico ou na Internet como um todo. Os outros recursos e conjuntos de informações disponíveis no dispositivo eletrônico são constituídos por 
outros softwares ou programas que codificam as possibilidades lógicas dos sistemas digitais, organizando-as para cumprir funções de calculadora, dicionário, bússola e tradutor de textos, por exemplo. Esses programas podem ter sido instalados no desenvolvimento do projeto original do dispositivo eletrônico ou podem ser posteriormente inseridos no sistema como aplicativos obtidos em repositórios de softwares acessados por meio da Internet.

A possibilidade de composição de um sistema multifuncional dentro do sistema lógico suportado pelo dispositivo eletrônico também permite a composição de ambientes multifuncionais, multimídia e hipertextuais, como um ambiente virtual de aprendizagem (AVA) que integra os e-books com outros diferentes recursos e conteúdos educativos.

\subsection{O e-book como fonte de informação e recurso didático}

As vinculações ou hiperlinks incorporados no formato e-book permitem que uma imagem ilustrativa do texto escrito seja um link para um hipertexto audiovisual previamente vinculado à estrutura do produto. Assim, ao ser acionada, a imagem passa a atuar como janela de projeção e atuação do hipertexto audiovisual. Para tanto, é necessário que o suporte eletrônico ou hardware disponha dos recursos necessários, incluindo, pelo menos, um software para configurar a informação em formato de hipertexto audiovisual. Mas, de acordo com o seu grau de sofisticação, o programa e-reader já é desenvolvido como software multifuncional.

No aspecto conceitual, entretanto, é preciso considerar como opção essa relação ou interação entre o conteúdo específico do e-book e as possibilidades informativas e interativas adicionais. Um conteúdo digital em formato de livro sem vinculações ou hiperlinks ainda é considerado e-book, apesar de não ser produto hipertextual.

Na perspectiva ampliada ou expandida, o e-book é um produto de conteúdo fechado, embora também seja vinculado por links aos recursos do dispositivo eletrônico, que lhe oferece suporte físico, e à multifuncionalidade ou à interfuncionalidade oferecida pelo software e-reader, que recebe e organiza a informação no formato de livro digital. 
A possibilidade hipermídia vinculada ao conteúdo específico do e-book proporciona o acesso a textos audiovisuais e, dessa maneira, viabiliza a inclusão de entrevistas, pequenas palestras e animações gráficas, com apresentação de modelos tridimensionais interativos, para serem visualizados em posições definidas pelo leitor. Há ainda os efeitos de zoom, que facilitam o alternar entre a visualização das imagens completas e o exame dos detalhes. O suporte digital permite a vinculação de dados geográficos com mapas interativos acessados por meio de sistemas de localização por satélite (GPS) (UNGARETTI; FRAGOSO, 2012).

É possível, portanto, considerar, de maneira ampla, que o e-book é uma fonte de conhecimento compilada em um único veículo constituído de conteúdos digitais, tais como: textos escritos, imagens fixas e dinâmicas e textos ou efeitos sonoros. Os conteúdos podem ser acessados online, através da Internet, ou offline, depois de terem sido carregados no dispositivo eletrônico (OWEN et al., 2008; BUFREM; SORRIBAS, 2009; DZIEKANIAK et al., 2010).

O e-book pode ser considerado um veículo de disseminação rápida de conteúdos, o que decorre da facilidade de atualização e correção do texto e do potencial colaborativo e interativo da comunicação em rede digital. Inclusive, pode proporcionar a interação dos leitores com os escritores do conteúdo informativo, dispensando a ação de intermediários nos processos de desenvolvimento e comercialização dos produtos. Quando o processo de produção e distribuição do $e$ book já é intermediado por uma editora, a Internet também amplia o acesso do consumidor final, oferecendo-lhe maior mobilidade e independência (BUFREM; SORRIBAS, 2009).

A utilização de e-books com finalidades didáticas se tornou uma prática recorrente na área de Educação. Há livros mais voltados para o público adulto, com conteúdo literário ou filosófico, em que as palavras escritas são os recursos necessários e suficientes para informar o leitor por intermédio de narrativa ou dissertação verbal. O livro didático adotado no ensino formal foi tradicionalmente utilizado em conjunto com o caderno de exercícios, que é, muitas vezes, incorporado ao próprio corpo do livro. Em geral, o livro didático requer ilustrações, mapas e gráficos como recursos auxiliares para ilustrar ou esclarecer as ideias do conteúdo ao leitor estudante. Além disso, nos livros cujo formato incorporou o caderno de 
exercícios, é comum a proposição de tarefas com espaços para sua realização e registro.

O e-book didático possibilita a atualização digital dos recursos ilustrativos e dos recursos para a realização das tarefas propostas, o que se ajusta ao perfil dos estudantes contemporâneos. Trata-se de estudantes da Geração Playstation, uma referência à marca de sistemas eletrônicos, incluindo hardware e software, para jogos, denominados videogames, com conteúdo dinâmico e audiovisual. Tais estudantes "[...] tendem a reagir melhor a aprendizagens baseadas em contexto interativo e dinâmico [...]" em sistema hipermídia (BIDARRA et al., 2012). Isso justifica a inserção dos e-books didáticos em ambiente virtual de aprendizagem (AVA) conectado à Internet.

\subsection{E-books didáticos}

O processo de ensino e aprendizagem baseado em suportes eletrônicos e mediação digital em rede, com o uso da Internet, é internacionalmente denominado de $e$ learning. Isso está inserido num processo mais amplo - que viabilizou a interação social com compartilhamento de informações por meio dos ambientes digitais comunitários e das redes sociais -, denominado inteligência coletiva.

O sistema de integração digital em rede que consolidou o atual estágio de interatividade digital é conhecido como Web 2.0. Há websites, como Wikipédia, YouTube e Flickr, que são exemplos de interatividade porque reúnem e disponibilizam, para toda a comunidade digital, conteúdos postados por iniciativa direta de seus membros. Também há a possibilidade de implementação de ambientes digitais coletivos, porém com acesso restrito aos membros previamente cadastrados, como é o caso do ambiente virtual de aprendizagem (AVA).

A Internet estabelece uma rede de websites e compõe o sistema de localidades digitais e canais que servem de ambiente para a postagem e a circulação dos e-books. Em princípio, um e-book didático é qualquer livro digital suportado por aparato eletrônico e usado para fins educativos. Entretanto, existem e-books que são especialmente produzidos como material didático e, de maneira geral, são caracterizados pelo incremento de recursos ilustrativos e interativos, com a 
finalidade de ilustrar e esclarecer o conteúdo verbal e propor exercícios para serem realizados pelo estudante. Desse modo, Jung (2009) conceitua o livro didático digital como um livro que, centrado no estudante, além de oferecer conteúdo verbal no formato de escritura, vincula as ideias propostas a diferentes possibilidades ilustrativas e interativas.

Vinculado a diferentes recursos e a múltiplas possibilidades complementares disponíveis no ambiente virtual de aprendizagem (AVA) ou na Internet, o uso do $e$ book e o acesso às bibliotecas virtuais permitem que o processo educativo junto ao estudante seja possível tanto no ambiente escolar quanto em casa. Isso supera os limites de tempo e espaço que restringem o acesso aos livros impressos e, ainda, conecta o estudo a diversos recursos hipermídia ou interativos, que são necessários à realização dos exercícios.

Juntamente com artigos jornalísticos ou acadêmicos, a relevância do $e$-book decorre, assim como a do livro em suporte de papel, da oferta de conteúdos completos e fechados, previamente organizados pelo autor para explicitar com palavras escritas determinado conteúdo. Já as informações disponíveis na Internet são, de maneira geral, fragmentadas e, muitas vezes, contraditórias. Dessa forma, em vez de informações esparsas, os e-books relatam as relações lógicas construídas no processo de produção do conhecimento.

A formatação Portable Document Format (PDF) é uma das maneiras de se configurar, apresentar e preservar o conteúdo integral do e-book. Com o uso da linguagem de marcação Extensible Markup Language (XML) é possível promover a hipertextualidade do documento por meio de vínculos ou hiperlinks com outros conteúdos. Para permitir a transferência dos dados em rede e garantir a integridade do formato em diferentes dispositivos, utiliza-se o padrão Sharable Content Object Reference Model (SCORM) para o conteúdo do objeto. As plataformas inseridas nos dispositivos eletrônicos para suportar os e-books didáticos precisam comportar diferentes sistemas operacionais, tais como: Windows, Linux, iOS, Android e softwares abertos (JANG, 2014).

É recomendável e oportuno que a plataforma de e-books didáticos seja hospedada em ambiente computacional nas nuvens, pois proporciona: (a) acesso ubíquo; (b) compartilhamento de dados e conteúdos; (c) redução de custos de 
licenças de softwares; (d) maior segurança dos dados; (e) prolongamento do ciclo de vida das máquinas; (f) disponibilidade de conectividade com a Internet fora das escolas; (g) alternativas de distribuição do tempo de aula; (h) fortalecimento da comunicação entre professor, escola, estudante e pais; (i) acesso dos estudantes, a partir de seus próprios computadores, à biblioteca virtual.

\section{Discussão e considerações}

Para que sejam evidenciadas as possibilidades e as vantagens que justificam o interesse pelo uso de e-books como parte dos investimentos nos processos educativos em ambientes de aprendizagem em rede, primeiramente, depende-se dos atendimentos das necessidades básicas de infraestrutura física e lógica, isto é, redes de computadores de banda larga, rede de energia, mobiliários e equipamentos adequados.

Além dos equipamentos e dos serviços disponíveis no espaço institucional, é imprescindível que os estudantes disponham de acesso a equipamentos e serviços em sua moradia e em outros espaços, porque isso possibilita o processo de aprendizagem em tempo integral. Há ainda interesse em que o sistema educativo envolva a participação dos pais dos estudantes e de outros membros da comunidade nesse processo. Por isso, além do acesso, é igualmente necessário que as pessoas que compõem a comunidade envolvida saibam utilizar, pelo menos, os recursos básicos da tecnologia digital. Os professores e tutores, por sua vez, devem dominar o uso dos recursos disponíveis, além dos conteúdos disciplinares do processo educativo.

As bibliotecas virtuais abrigadas em repositórios nas nuvens viabilizam o acesso a distância aos e-books, permitindo aos estudantes consultar, identificar, capturar, ler e interagir com o conteúdo informativo, desde que estejam em área de acesso à Internet. Além de escolher os e-books, os estudantes podem armazená-los na memória de seu dispositivo eletrônico para acessá-los e consultá-los onde quer que estejam.

Considerando-se as informações e os argumentos propostos por Ash (2011), Embong e outros (2012) e Jang (2014), é possível elencar as possiblidades e as 
vantagens decorrentes do uso de $e$-books didáticos vinculados a sistemas educativos ou ambientes virtuais de aprendizagem (AVA):

a) adaptação ou personalização de processos interativos e conteúdos, de acordo com as características individuais do estudante: os recursos do sistema podem oferecer conteúdo coerente com o estilo e o nível do estudante. Dessa forma, não é necessário propor $e$-books ou programas de atividades iguais para todos. Além disso, o conteúdo do e-book pode ser vinculado a outros recursos hipermídia e conteúdos multimídia, variando as possibilidades de ilustração, esclarecimento, complementação e prática educativa. Ainda, devido à flexibilidade resultante das possibilidades integradas do suporte eletrônico e do programa e-reader, o leitor estudante pode variar o tamanho das letras do texto escrito ou requerer que o mesmo seja lido em um determinado idioma, inclusive regulando o volume de som da voz, entre outras possibilidades;

b) amplitude no tempo e no espaço da comunicação e da interação entre estudantes e outros envolvidos: os recursos para a comunicação interativa oferecida pelo sistema de aprendizagem que integra e conecta os $e$-books permitem a cooperação síncrona e assíncrona entre os estudantes e também a comunicação com professores e tutores do processo educativo, inclusive podendo envolver os pais ou outros responsáveis pela educação dos estudantes. Caso os limites éticos permitam registros do processo de desenvolvimento do estudante, as atividades e avaliações podem ser acessadas e acompanhadas pelos responsáveis;

c) eficiência e agilidade na gestão do processo educativo: pode haver vínculos entre o conteúdo do e-book e o registro dos exercícios de treinamento ou avaliação. Isso oportuniza um sistema de gerenciamento da aprendizagem por meio do qual o desempenho dos estudantes é considerado de maneira ampla, tendo em vista sua presença na sala de aula, o resultado de avaliações pontuais e, também, todo o seu percurso didático, porque há o registro detalhado de todos os passos do processo educativo, com data e hora. O próprio sistema pode identificar e avisar sobre procedimentos incorretos e, desse modo, possibilitar a revisão da 
tarefa por parte do estudante e da proposta por parte do professor e de outros envolvidos no planejamento do projeto pedagógico. Tudo isso torna possível alguns procedimentos personalizados e, em uma proposta radicalmente diferenciada, o estabelecimento de um percurso de aprendizagem adaptado a cada estudante.

d) redução dos custos pessoais, financeiros e ambientais: o volume dos materiais transportados por estudantes e professores é reduzido quando o processo educativo ocorre com recursos eletrônicos. O transporte de livros, cadernos de registro e de atividades ou equipamentos, como calculadora, régua e outros, pode ser resumido a um dispositivo eletrônico móvel. Portanto, reduz-se o esforço mental e físico do estudante e do professor para lembrar-se dos materiais necessários e, depois, reuni-los e transportá-los até a sala de aula. No âmbito financeiro, o aumento de escala na produção e nas vendas de equipamentos eletrônicos e sistemas digitais torna os preços menores e os produtos mais acessíveis. Mesmo sem considerar todos os recursos e os conteúdos já disponíveis na Internet, uma instituição de ensino pode manter contratos com empresas fornecedoras de equipamentos e softwares e com distribuidoras de conteúdo em formato de e-book para oferecer acesso aos estudantes a esses produtos sem que haja a necessidade da compra de livros impressos e outros materiais no início de cada ano letivo. Os recursos necessários para todas as séries estarão disponíveis no ambiente educativo digital e o custo de acesso já estará incluído na mensalidade paga pelos estudantes. Apesar da expectativa generalizada de que a cultura digital promova de maneira evidente a redução dos custos ambientais, isso ainda depende de circunstâncias mais amplas: por exemplo, investimentos na produção de energia limpa, na coleta e no tratamento responsável da sucata eletrônica e no contínuo avanço tecnológico para reduzir os sistemas físicos de suporte dos dados digitais.

Por tudo que foi apresentado neste artigo, considera-se que o esforço para ampliar a aplicação de aprendizagem em rede, apoiada no uso de $e$-books didáticos, é recompensado com: (1) economia de tempo; (2) ampliação dos recursos 
educativos, com mais informações e mais instrumentos multimídia e hipermídia; (3) incremento da comunicação, da gestão e da autogestão do processo educativo; e (4) acesso a distância aos recursos didáticos necessários ao estudo, entre outros benefícios.

As possibilidades e vantagens justificam o investimento institucional, profissional e pessoal para reunir as condições necessárias à consolidação dos ambientes de aprendizagem em rede como apoio ao ensino presencial ou como prática educativa a distância. Isso requer uma preparação antecipada, que é ampla e trabalhosa, e deve ser executada por gestores, técnicos e educadores envolvidos, e realizada com todo o rigor técnico e científico possível.

De modo efetivo ou precário, a utilização de e-books e de outros recursos digitais no processo de aprendizagem está consolidada. Entretanto, é cada vez maior a necessidade de organização e gestão eficientes dos sistemas formais ou informais dos ambientes de aprendizagem em rede, porque é preciso conhecimento e competência para que os benefícios decorrentes da prática educativa online atinjam a plena eficiência na formação pessoal e social.

\section{Referências}

ASH, K. Publishers Turn to Cloud Computing to Offer Digital Content. Education Week, Bethesda, v. 5, n. 1, p. 34-36, 2011.

BIDARRA, J. et al. O gamebook como modelo pedagógico: investigação e desenvolvimento de um protótipo para iPad, In: Carvalho, A. Aprender na era digital: jogos e mobile-learning. Santo Tirso: DeFacto, 2012.

BOTTENTUIT, J. B.; COUTINHO, C. P. A problemática dos e-books: um contributo para o estado da arte. In: CONFERENCIA IBERO-AMERICANA EM SISTEMAS, CIBERNÉTICA E INFORMÁTICA (CISCI), 6., 2007, Orlando, EUA. Anais... Orlando, 2007.

BUFREM, L.; SORRIBAS, T. Práticas de leitura em meio eletrônico. ETD Educação Temática Digital, Campinas, v. 11, n. 1, p. 298-326, dez. 2009. Disponível em: 〈http://www.fe.unicamp.br/revistas/ged/etd/article/view/2038>. Acesso em: 08 out. 2014.

CAVALCANTE, A. et al. A iconografia em comunidades indígenas. Revista 
Projética, Londrina, v.4, n.2, p. 09-28, jul/dez., 2013. Disponível em: <http://www.uel.br/revistas/uel/index.php/projetica/article/viewFile/16043/14237>. Acesso em: 08 out. 2014.

CERVO, A. L.; BERVIAN, P. A.; SILVA, R. Metodologia científica. 6. ed. São Paulo: Pearson Prentice Hall, 2007.

DZIEKANIAK, G. et al. Considerações sobre o e-book: do hipertexto à preservação digital. Biblos: Revista do Instituto de Ciências Humanas e da Informação, Rio Grande, v. 1, n. 2, p. 83-99, jul./dez. 2010. Disponível em:

<http://www.seer.furg.br/biblos/article/view/1899/1035>. Acesso em: 08 out 2014.

EMBONG, A. M. et al. E-books as textbooks in the classroom. Procedia - Social and Behavioral Sciences, Louisville, v. 47, p. 1802-1809, 2012.

FEDERAL COMMUNICATIONS COMMISSION - FCC. Digital textbook playbook: the digital textbook collaborative. 2012. Disponível em:

$\langle$ http://www.fcc.gov/encyclopedia/digital-textbook-playbook>. Acesso em: $30 \mathrm{abr}$. 2014.

GROUPE SPECIALE MOBILE - GSMA. Mobile education landscape report. Londres, 2011. Disponível em: 〈http://www.gsma.com/connectedliving/wpcontent/uploads/2012/03/landscape110811interactive.pdf>. Acesso em: 17 jul. 2014.

JANG, S. Study on service models of digital textbooks in cloud computing environment for SMART education. International Journal of u-and e-Service, Science and Technology, Tasmânia, v. 7, n. 1, p.73-82, 2014. Disponível em: <http://www.sersc.org/journals/IJUNESST/vol7_no1/7.pdf>. Acesso em: 19 ago. 2014.

JUNG, S. M. Leading future education: development of digital textbooks in Korea. In: WORLD TEACHERS' DAY IN THAILAND, 4., 2009, Bangkok; UNESCOAPEID INTERNATIONAL CONFERENCE QUALITY INNOVATIONS FOR TEACHING AND LEARNING, 12., 2009, Bangkok. Anais... Bangkok, 2009.

OWEN, V. et al. E-books in research libraries: issues of access and use. Ontário, Canadá: CARL, 2008. Disponível em: <http://carl-

abrc.ca/uploads/pdfs/copyright/carl_e-book_report-e.pdf>. Acesso em: 17 jul. 2014.

PERASSI, R.; MENEGHEL, T. Conhecimento, mídia e semiótica na área de Mídia do Conhecimento. In: VANZIN, T.; DANDOLINI, G. (Org.). Mídias do conhecimento. Florianópolis: Padion, 2011. v. 1.

PROCÓPIO, E. O livro na era digital: o mercado editorial e as mídias digitais. São Paulo: Giz, 2010.

United Nations Educational, Scientific and Cultural - UNESCO. The future of mobile learning: implications for policy makers and planners. Paris: UNESCO, 
2013. Disponível em:

<http://unesdoc.unesco.org/images/0021/002196/219637E.pdf>. Acesso em: 15 jul. 2014.

UNGARETTI, C. E.; FRAGOSO, S. Design gráfico para $e$-books e livros impressos: proposta de método de projeto simultâneo para explorar a complementaridade dos suportes. Educação Gráfica, Bauru, v. 16, n. 3, p. 17-33, 2012.

\title{
E-textbooks in networked learning environments
}

\begin{abstract}
This article presents a study of the relationship between e-books and virtual learning environments (VLE) supported by electronic devices connected to the Internet. The objective is to highlight the opportunities and advantages that justify the interest in the use of e-books as part of the investments in education processes in networked learning environments. In order to do so, an exploratory bibliographic research with qualitative bias was performed. As possibilities and advantages of using e-textbooks, we have listed: the adaptation or customization of interactive processes and content according to the individual characteristics of the student; the increased range in time and space for communication and interaction between students and other participants; efficiency and agility in the management of the educational process; and the reduction of personal, financial and environmental costs. However, for that to happen, we depend on meeting the needs of the physical and logical infrastructure, that is, broadband Internet, power grid, furniture and appropriate equipment.
\end{abstract}

Keywords: E-textbooks. Virtual Learning Environment. Educational technology. Elearning.

Recebido: 08/10/2014

Aceito: 16/03/2015 